\title{
Seeing the Forest and the Trees: Default Local Processing in Individuals with High Autistic Traits Does Not Come at the Expense of Global Attention
}

\author{
Ryan A. Stevenson ${ }^{1} \cdot$ Sol Z. Sun ${ }^{1,2} \cdot$ Naomi Hazlett $^{1} \cdot$ Jonathan S. Cant $^{2} \cdot$ \\ Morgan D. Barense ${ }^{1,3} \cdot$ Susanne Ferber ${ }^{1,3}$
}

(C) Springer Science+Business Media New York 2016

\begin{abstract}
Atypical sensory perception is one of the most ubiquitous symptoms of autism, including a tendency towards a local-processing bias. We investigated whether local-processing biases were associated with global-processing impairments on a global/local attentional-scope paradigm in conjunction with a composite-face task. Behavioural results were related to individuals' levels of autistic traits, specifically the Attention to Detail subscale of the Autism Quotient, and the Sensory Profile Questionnaire. Individuals showing high rates of Attention to Detail were more susceptible to global attentional-scope manipulations, suggesting that local-processing biases associated with Attention to Detail do not come at the cost of a global-processing deficit, but reflect a difference in default global versus local bias. This relationship operated at the attentional/perceptual level, but not response criterion.
\end{abstract}

Keywords Autism spectrum disorder - Face recognition . Sensory processing · Composite-face effect · Vision . Attention $\cdot$ Global processing $\cdot$ Local processing

\section{Introduction}

Visual objects are typically composed of component features that together uniquely identify the object. With typical visual processing, an individual tends to perceive the

Ryan A. Stevenson

ryan.andrew.stevenson@gmail.com

1 Department of Psychology, University of Toronto, 100 Saint George Street, Toronto, ON M5S 3G3, Canada

2 Department of Psychology, University of Toronto Scarborough, Toronto, ON, Canada

3 Rotman Research Institute at Baycrest, Toronto, ON, Canada object as a unified whole, demonstrating a global attentional scope (Eriksen and James 1986; Müller et al. 2003; Rauschenberger and Yantis 2001). A classic example of this is face perception. When viewing a face, one does not typically notice two eyes, a mouth, and a nose, but instead perceives a unitary face, bound as an integrated representation (Maurer et al. 2002; Rossion 2013; Young et al. 1987). Whereas most individuals can consciously alter their visual scope to the local level, focusing on the eyes, for example, the default mode of attention is at the global level, known as a global-processing bias (Hughes et al. 1984; Navon 1977, 1981).

Autism spectrum disorder (ASD), however, has been theorized to be associated with a reduction in global-processing bias, or even a local-processing bias. For instance, Kanner's original description of autism (Kanner 1943), the weak central coherence hypothesis (Frith and Happe 1994), and more recent theories such as the predictive coding hypothesis (Pellicano and Burr 2012), all include plausible explanations for such a local-processing bias. Further evidence in support of the idea that there is an altered default processing style in ASD can be seen in the plethora of studies reporting that autistic individuals ${ }^{1}$ show intact or even enhanced local-oriented perception-often outperforming typically developed on tasks where local processing is the optimal strategy (Bertone et al. 2005; Happé

\footnotetext{
1 There is some debate as to whether person-first language should be used in describing autistic individuals (e.g. "individuals with autism" vs. "autistic individuals"). While researchers and clinicians often support the use of person-first language, recent studies have shown that autistic individuals themselves, their families and their caregivers, most commonly prefer language that incorporates autism as a component of their identity (Kenny et al., 2015). As such we will use the language preferred by autistic individuals themselves throughout this manuscript.
} 
1996; Jolliffe and Baron-Cohen 1997; Joseph et al. 2009; Kemner et al. 2008; Minshew et al. 1997; O'Riordan and Plaisted 2001; Shah and Frith 1983, 1993).

Theories of local-processing bias in ASD often suggest that this increase in local processing comes at the cost of global processing. Whereas a number of studies have indeed found a decrement in global processing (Behrmann et al. 2006a; Bölte et al. 2007; Pellicano et al. 2005; Plaisted et al. 1999; Rinehart et al. 2000), a similar number of other studies have shown intact global-processing abilities in ASD (Deruelle et al. 2006; Mottron et al. 2003, 2006; Wang et al. 2004) or intact global processing at the group level in the presence of differences in processing strategies (Johnson et al. 2010). There are a number of factors that might contribute to these mixed results, but one reason worth noting is that despite the widespread usage of the terms "global" and "local" processing, it is not the case that all tasks assessing global and local processing in fact measure the same underlying cognitive processes. For example, visual search tasks (O'Riordan et al. 2001; O'riordan 2004), embedded figures tasks (Jolliffe and Baron-Cohen 1997; Shah and Frith 1983), and change detection tasks (Gomot et al. 2002, 2006) have all been shown to have intact or enhanced processing in ASD, but draw on significantly different cognitive processes from one another. Given a similar pattern of results, however, performance on all of these tasks is interpreted as reflecting "local" processing. The same can be said for studies that focus on "global" processes-autistic individuals have been shown to have reduced perception of global motion (Pellicano et al. 2005) but increased global integration of contour (Almeida et al. 2014). Whereas discrepancies these could be discussed in terms of mixed results with regard to global processing in autism, they may also arise from the fact that motion perception and integration of contour are subserved by different underlying neuro-cognitive operations. Given this ambiguity, and its potential for generating mixed or discrepant results, we will explicitly define local processing as the attentional or sensory preference for the fine-grain detail of a sensory input, and global processing as the attentional or sensory preference for the course-grain detail of a sensory input.

In addition to the aforementioned issues in studying global and local processing across studies utilizing different paradigms, results also vary within studies that employ the same paradigms. For example, Mottron et al. (2003) presented participants with composite letters (Fig. 1; Navon 1977) in which large letters (global) were comprised of smaller letters (local). Participants made speeded responses to identify a target letter that could appear in either the larger, global letter or the smaller, local letters. Autistic individuals showed no difference from typical controls in response times during the detection task, regardless of whether the target was presented in the global or local letters, suggesting that global processing is not compromised in autism, but is actually intact (Mottron et al. 2003, 2006).

In a similar detection study (Plaisted et al. 1999), attentional scope was manipulated, with two selective-attention conditions: one in which targets were presented at the local level, and a second in which targets were presented at the global level. Importantly, a divided-attention condition was also presented in which targets could be presented at either the global or local levels. When attentional scope was specifically directed to one level or the other, autistic and typically-developed individuals both showed similar performance patterns, with faster responses in the global-attention than in the local-attention condition. However, in the undirected, divided-attention condition, autistic individuals made more errors when the targets were in the global letters, whereas the typically developed individuals made more errors when the targets were in the local letters. These findings, taken together, help to further constrain earlier hypotheses, such that whereas autistic individuals default to local processing at the expense of global processing, their global-processing abilities are not diminished when their attentional scope is explicitly directed towards attending globally (Koldewyn et al. 2013; Van der Hallen et al. 2014).

In addition to the composite-letter tasks described above, global and local processing can also be measured through the composite-face effect (CFE; Cheung et al. 2008; Young et al. 1987). In the composite-face task, participants are asked to judge if the top halves of two sequentially presented faces are the same or different, while ignoring the bottom halves of the faces. Importantly, the faces are chimeric, in which the top and bottom halves of the faces vary independently, allowing for congruent and incongruent trials (Fig. 1). Due to the holistic nature of face perception, the typical observer is unable to entirely ignore the bottom halves. Thus two identical top halves will look different when the bottom halves differ (incongruent condition), because the different bottom halves influence the perception of the top halves. The second key component of the composite-face task is that when the top and bottom halves of the faces are offset, or misaligned, performance recovers and the effect is drastically diminished, as the faces are no longer perceived as a unified whole (Fig. 1a, right). The disproportionate impact of congruency when aligned, relative to when misaligned, is referred to as the composite-face effect.

Given that the CFE is influenced by a global-processing bias (Busigny and Rossion 2011; Gao et al. 2011), and following the hypothesis that autistic individuals show a reduction in global-processing bias or even a local-processing bias (Frith and Happe 1994), one would predict that 
Fig. 1 Experimental protocol. a An example trial for an aligned composite-face trial. b An example of a misaligned composite-face trial. c For the composite-letter task,

individuals were cued to attend either the global or local letters, and made a same different judgment as to whether the letters at the attended level were the "same" or "different". d For the composite-face task, participants were asked to make a same-different judgment on only the top halves of the faces. In "congruent" trials, the unattended bottom halves of the faces led to the same response as the attended, top halves of the faces, whereas in "incongruent" trials, unattended bottom halves of faces led to the opposite response as the attended top halves of the faces
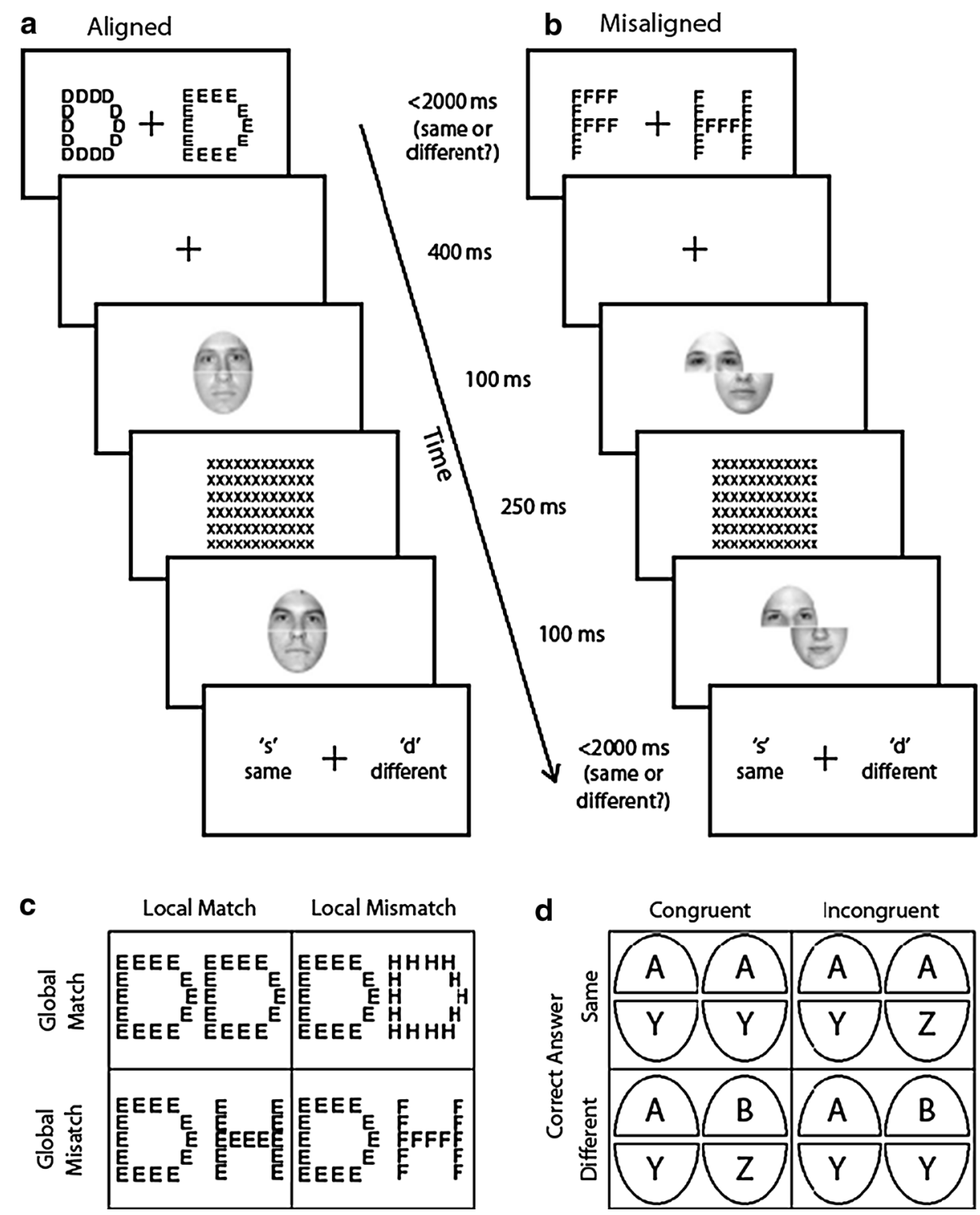

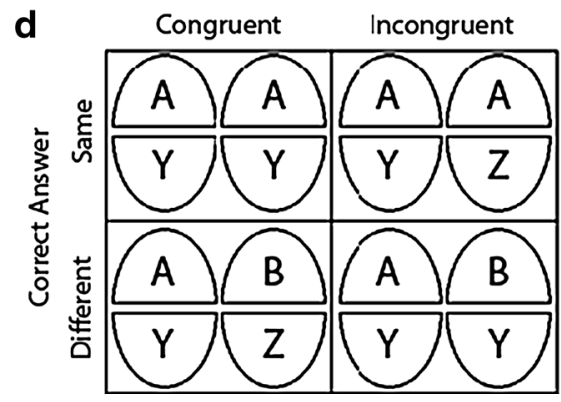

autistic individuals should show a weaker CFE than their typically developing peers. However, as with the aforementioned studies investigating global-processing deficits using composite letters, the evidence here has also been mixed. A recent study investigating the CFE in autistic children reported a reduction in the magnitude of the CFE relative to typically developed controls (Gauthier et al. 2009), while other studies find no evidence of such a reduction (Teunisse and de Gelder 2003). Additionally, Nishimura et al. (2008) reported that autistic adults exhibit a CFE that is on par with typically developed individuals, although the difference did trend towards significance $(p=0.12)$ with a relatively small sample size $(\mathrm{n}=17)$, reflecting a reasonable effect size $\left(\eta_{p}^{2}=0.08\right)$. A possible explanation for these discrepancies is a difference in sample demographics and phenotype, which vary drastically from study to study. For example, Gauthier et al. (2009), which found impaired global processing, studied children averaging 12 years of age, while Teunisse and de Gelder (2003) and Nishimura et al. (2008) studied autistic adults (mean age $=19.5$ and 20.6 years old, respectively).

Beyond differences in mean age of the samples, ASD itself is extremely heterogeneous, which may also account for these discrepancies in study results. From either the classic view of ASD as a cluster of disorders (Autism Disorder, Asperger's Disorder, and Pervasive Developmental Disorder-Not Otherwise Specified; APA 2000), or the newer spectrum of ASD from mild to severe (APA 2013), any two individuals with an ASD diagnosis can present with drastically different symptoms. These individual differences can include variability in global/local 
processing bias, which may be the source of such discrepant findings in simple group average results. A number of measures have been developed recently in an attempt to account for these individual differences in groups with ASD, including the measure we use here-the Autism Quotient (AQ; Baron-Cohen et al. 2001a, b). Using such measures to isolate different symptomatologies in the ASD phenotype allows one to explore what relationship a specific ASD trait has with a given experimental result. Furthermore, as a spectrum disorder, specific traits associated with ASD are not only found in individuals with a clinical diagnosis, but are also found in the general population. As such, studying these ASD traits in non-clinical samples provides a practical way to explore the relationship between specific aspects of the autism spectrum that may otherwise not be feasible. Moreover, a second measure that may account for phenotypic variability is the Sensory Profile (SP; Brown et al. 2001; Dunn and Westman 1997). Atypical sensory processing is now included as a diagnostic feature in ASD, and the SP has been developed to characterize sensory issues commonly found in ASD, including sensory hyper/hyposensitivity and sensory seeking and avoiding behaviors. The SP has also been successfully correlated with individuals' behavioral performance on visual perception tasks requiring global processing (Lowe et al. 2015).

In the current study, we tested for individual differences in ASD traits specifically related to global/local attentional scope in a non-clinical sample of adults. To explore how different processing styles relate to ASD traits we manipulated attentional scope by asking individuals to attend to either the global or local level in a composite-letter task. We then tested for the effect of this modulation on the composite-face task. In line with past studies, we hypothesized that inducing a global processing bias using the composite-letter task would result in an increase in the magnitude of the CFE, and conversely, inducing a local processing bias would result in a decrease of the CFE (Gao et al. 2011). In addition, participants completed questionnaires measuring ASD traits (AQ; Baron-Cohen et al. 2001a, b) and stable sensory processing characteristics (Sensory Profile, SP; Brown et al. 2001; Dunn and Westman 1997). We hypothesized that individuals showing higher levels of ASD traits in general would be more biased towards local processing. More specifically, we predicted that high scores on the Attention to Detail subscale of the Autism Quotient (Baron-Cohen et al. 2001a, b; Stewart and Austin 2009) would be predictive of localprocessing biases. Finally, given our prediction that local processing bias is a default processing style in ASD not associated with global-processing deficits (Mottron et al. 2003, 2006; Plaisted et al. 1999), we expected individuals with high autistic traits to show stronger global than local attentional scope effects. That is, if it is indeed the case that individuals higher in ASD traits tend to default to a localprocessing bias, then global attentional scope instructions may cause a greater shift in perceptual bias relative to those whose default attentional scope already tends toward global processing.

\section{Materials and Methods}

\section{Participants}

Participants included 48 undergraduate students (37 female; 44 right handed; mean age: 20.6 years, $\mathrm{SD}=1.84$, range: $18-28$ years) at the University of Toronto. All participants reported normal or corrected-to-normal vision, had no history of psychological or neurological disorders, and were given course credit for participation. All protocols were approved by the University of Toronto Research Ethics Board.

\section{Stimuli}

Composite-letter stimuli (presented in pairs; see Fig. 1) consisted of black composite letters (Navon 1977) on a white background, presented to the left and right side of a central fixation cross. Each local letter element was $0.2 \mathrm{~cm}$ wide $\times 0.3 \mathrm{~cm}$ high $\left(0.19^{\circ} \times 0.28^{\circ}\right)$. Each global letter was $1.0 \mathrm{~cm}$ wide $\times 1.5 \mathrm{~cm}$ high $\left(0.96^{\circ} \times 1.43^{\circ}\right)$. Control stimuli consisted of solid letters (as opposed to composite letters), where each letter was $1 \mathrm{~cm}$ wide $\times 1.5 \mathrm{~cm}$ high $\left(0.96^{\circ} \times 1.43^{\circ}\right)$. The center of each letter was $4.5 \mathrm{~cm}$ to the left and right of fixation. The letters were presented in black Helvetica bold font, and consisted of "D," "E," "F," and " $\mathrm{H}$ " in all possibilities of their local and global combinations, making 16 distinct composite letter stimuli.

Composite-face stimuli (presented sequentially; see Fig. 1) consisted of greyscale faces from http://agingmind. utdallas.edu/facedb. Using 96 (48 male, 48 female) original face images, 288 unique composite faces were created (top/ bottom pairs). For each of these 288, there was an aligned version and a misaligned version (576 total images). The face tops and bottoms were randomly paired (gender was always matched), and the same pairings were used for every participant. When aligned, stimuli were $2 \mathrm{~cm}$ wide $\times 3 \mathrm{~cm}$ tall $\left(1.91^{\circ} \times 2.86^{\circ}\right.$ visual angle $)$. The top and bottom halves of the misaligned faces were offset by $1 \mathrm{~cm}$, resulting in a stimulus $3 \mathrm{~cm}$ wide $\times 3 \mathrm{~cm}$ tall $\left(2.86^{\circ} \times 2.86^{\circ}\right.$ visual angle $)$. Within each trial, each first presentation of a composite-face stimulus was followed by a centrally presented mask to avoid aftereffects. Masks were $4 \mathrm{~cm}$ wide and $3.5 \mathrm{~cm}$ tall $\left(3.82^{\circ} \times 3.34^{\circ}\right.$ visual angle) and consisted of an array of Xs, with each individual 
" $\mathrm{X}$ " being $0.2 \mathrm{~cm}$ wide $\mathrm{x} 0.3 \mathrm{~cm}$ tall $\left(0.19^{\circ} \times 0.29^{\circ}\right.$ visual angle).

\section{Experimental Procedure}

Procedures were similar to that of Gao et al. (2011), who successfully utilized the composite-letter task to induce changes in the CFE. Participants were seated in a quiet room approximately $60 \mathrm{~cm}$ away from a computer screen and were instructed that they would view pairs of letter images on the screen. They were provided with an example of a pair of composite letters (the letter "H" composed of different local elements on either side of fixation, the letter "D" on one side and the letter "E" on the other-see Fig. 1 for examples). Participants were asked to respond as to whether the two letters were the same or different, with their focus on either the big (global) or the small (local) letters. In the example presented with the instructions, the answer would be "same" if they were focusing on the big, global letters, but "different" if they were focusing on the small, local letters. Participants were then told that following each letter trial, they would see a pair of images of faces, one after another, and that their job was to decide whether the top halves were the same or different. An example was presented where the top halves were different, but the bottom halves were the same (incongruent), and they were instructed that the correct answer should be "different." Finally, participants were shown a pair of misaligned faces and told that regardless of whether or not the faces were aligned, their task was still the same, namely to identify whether the top halves of the faces were the same or different.

Individual trials consisted of a single composite-letter prime followed by a composite-face task presentation (Fig. 1a). Each trial began with a $400 \mathrm{~ms}$ fixation screen, followed by a presentation of a pair of composite letters (or in the control condition, a pair of block letters), one on each side of the fixation cross, ending at either $2000 \mathrm{~ms}$ after onset or when an "s" (for same) or " $d$ " (for different) button press was made by the participant. The compositeface task of each trial began with a $400 \mathrm{~ms}$ fixation, followed by a $100 \mathrm{~ms}$ presentation of the study face, a $250 \mathrm{~ms}$ mask, and a $100 \mathrm{~ms}$ presentation of the test face. It should be noted here that these presentation times differ from Gao et al.'s study, where presentation times were longer. This divergence was introduced to avoid ceiling effects in order to attain sufficient between-subject variability to correlate with questionnaire measures. Finally, a response screen with "same or different" was displayed, ending at either 2000 ms or when an "s" or "d" button press was made by the participant.

The experiment was broken into three blocks to manipulate attentional scope: globally-primed, locally- primed, and control. Participants were instructed to attend to either the global, local, or single letter (control) at the start of each block. In each block, 256 trials were presented. Within each block of 256 trials, half of the composite-letter stimuli pairs were selected such that the letter(s) in the unattended level (e.g., attending to local elements under global instructions) would lead to a different answer than the letter(s) in the attended level. Half of the composite-face presentations were aligned, with an equal number of congruent (where the unattended, bottom portion of the face could lead to the correct answer) and incongruent presentations. Within each block, the correct answer of "same" (with the two top halves of the faces being identical) was equally likely as the correct answer of "different." Assignment of face stimuli to conditions (aligned vs. misaligned; congruent vs. incongruent) was counterbalanced across participants using a Latin square design, and block orders were counterbalanced across participants.

\section{Questionnaires}

All participants completed the Autism Quotient Questionnaire (AQ; Baron-Cohen et al. 2001a, b), a 50-item, selfreport measure commonly used to measure traits associated with ASD. Items were originally grouped into five subcategories; Social Skills, Communication, Attention to Detail, Attention Switching, and Imagination. Subsequent data-driven factor analyses have identified between two and four subcategories, including combinations of Socialness, Attention to Detail, Communication, and Imagination (Hoekstra et al. 2008; Hurst et al. 2007; Stewart and Austin 2009), though naming schemes vary between reports.

Finally, sensory processing style was assessed using the four-quadrant 60-item Adult Sensory Profile (Brown et al. 2001; Dunn and Westman 1997), which measures stable sensory preferences such as sensory seeking/avoiding and overall level of sensory sensitivity. Participants indicated a response on a five-point Likert scale (almost never; seldom; occasionally; frequently; almost always).

\section{Results}

\section{Survey Measures}

\section{Autism Quotient}

Individuals' autism quotients were scored as outlined in Baron-Cohen et al. (2001a, b) original report of the measure. Participants' scores ranged from 4 to 33, with a group mean of 16.6 and standard deviation of 5.8. Scores of 32 and above $(n=1)$ are considered at risk for clinical ASD. 
Subscales were isolated according to Stewart and Austin's (2009) large-scale, data-driven factor analysis of the AQ which resulted in four distinct subscales, Socialness, Attention to Detail, Understanding others/Communication, and Imagination. Scores on these subscales were correlated with individuals' performance on the composite letter and face tasks, reported below. Of primary relevance here is the Attention to Detail subscale, (AQ items 5, 6, 9, 12, 19, 23, 29, and 41) which focuses on fine-grain details and patterns, and is thus relevant to local processing biases. Example items include "I tend to notice details that others do not" and "I often notice small sounds when others do not."

\section{Sensory Profile}

Participants' scores on the Adult Sensory Profile, measuring stable sensory preferences, were scored using methods outlined in the published manual (Brown and Dunn 2002). Scores ranged from 111 to 209 , with a mean of 162.7 and a standard deviation of 18.4 .

\section{Composite-Letter Task: Accuracy}

Mean accuracies for each of the three levels of attentional scope on the composite-letter task (control, global, and local) were calculated for each participant. We observed mean accuracies of $94.2 \%$ (standard error $=0.7 \%$ ) for the control condition, $93.6 \%$ (standard error $=0.6 \%$ ) for the global attentional scope condition, and $90.6 \%$ (standard error $=1.6 \%$ ) for the local attentional scope condition (Fig. 2). A one-way repeated-measures analysis of variance (ANOVA) with the within-subject factor of attentional scope (control, global, local) revealed a significant main effect of scope $\left(F_{(2,94)}=5.47, p=0.006\right.$, $\left.\eta^{2}=0.10\right)$. Pairwise follow-up t-tests revealed significant differences between the control and local attentional scope

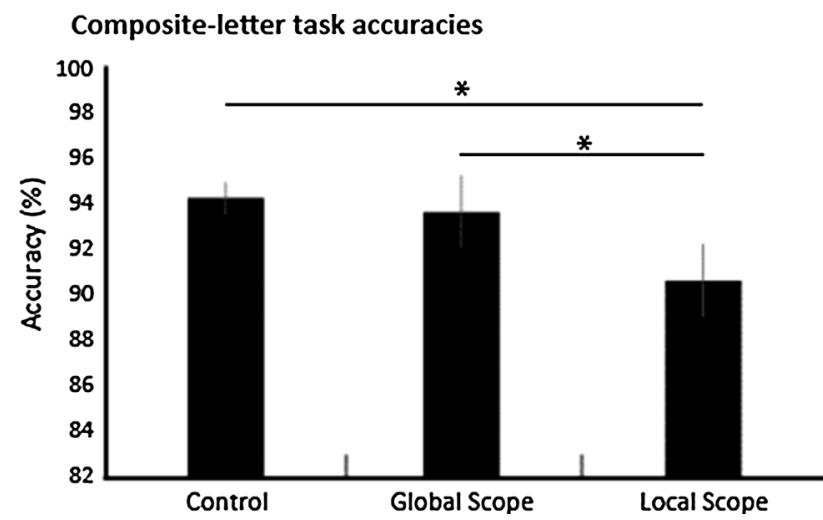

Fig. 2 Composite-letter task. Response accuracies to the compositeletter task, which was used to manipulate attentional scope towards global or local processing. Asterisk Indicates significant differences at the level of $p<0.05$ $\left(t_{(47)}=2.68, p=0.01, d=0.42\right)$, between the global and local attentional scopes $\left(t_{(47)}=2.12, p=0.04, d=0.36\right)$, but not between the control and global attentional scope $\left(t_{(47)}=1.10, p=0.28, d=0.12\right)$. Thus, the significant main effect of accuracy in the composite-letter task was driven by a decrease in accuracy during the local-processing but not global-processing condition, relative to control, consistent with previously reported "globalprecedence" effects (Gao et al. 2011; Navon 1977).

Given this significant difference between global and local processing, global-precedence scores were calculated for each individual by subtracting each individual's mean accuracy during the local attentional scope manipulation from their mean accuracy during the global attentional scope manipulation. As such, positive values indicate a global precedence. Global precedence at the group level was observed at $3.0 \%$ (standard error $=1.4 \%$ ), and was seen in 31 of 48 participants, significantly greater than the number that would be due to chance (binomial $p=0.015$ ).

\section{Composite-Face Task: Sensitivity (Az)}

Performance on the composite-face task was indexed using Az, a signal detection measure (cf. Gao et al. 2011; Verde et al. 2006). Az is a monotonic transformation of d' bounded by 0 and 1 , with the advantage that $\mathrm{Az}$ is less susceptible to response bias than d' (Richler et al. 2008; Verde et al. 2006). An omnibus three-way repeated-measures ANOVA was conducted with factors of attentional scope (control, global, and local), alignment (aligned and misaligned), and congruency (congruent and incongruent). This analysis revealed main effects of alignment $\left(\mathrm{F}_{(1,47)}=20.5, \mathrm{p}<0.001\right.$, $\left.\eta_{\mathrm{p}}^{2}=0.30\right)$ and congruency $\left(\mathrm{F}_{(1,47)}=125.5, \mathrm{p}<0.001\right.$, $\left.\eta_{\mathrm{p}}^{2}=0.73\right)$, as well as an alignment $\mathrm{x}$ congruency interaction $\left(F_{(1,47)}=105.9, p<0.001, \eta_{p}^{2}=0.69\right)$. Follow up tests, collapsing across attentional scopes, revealed that this effect was driven by a strong difference in sensitivity between congruent and incongruent presentations that were aligned $\left(t_{(47)}=12.27, p=2.88 \mathrm{e}^{-16}, \mathrm{~d}=1.26\right)$ relative to a small difference when misaligned $\left(t_{(47)}=2.27, p=0.03\right.$, $\mathrm{d}=0.13$ ), representing the classic CFE (Fig. 3a).

As Fig. 3b, c illustrate, there was no main effect of attentional scope $\left(\mathrm{F}_{(2,46)}=1.62, p=0.20, \eta_{\mathrm{p}}^{2}=0.03\right)$, no interaction between attentional scope and alignment $\left(\mathrm{F}_{(2,46)}=1.42, \quad p=0.25, \eta_{\mathrm{p}}^{2}=0.03\right)$ or congruency, $\left(\mathrm{F}_{(2,46)}=1.81, p=0.17, \eta_{\mathrm{p}}^{2}=0.04\right)$, and no three way interaction $\left(\mathrm{F}_{(2,46)}=0.15, p=0.86, \eta_{\mathrm{p}}^{2}<0.01\right)$.

We also directly compared the strength of the CFE for each individual, under each attentional scope (Fig. 3c). Strength of the CFE was calculated as:

$$
\begin{aligned}
& \left(A z_{\text {aligned congruent }}-A z_{\text {aligned incongruent }}\right) \\
& \quad-\left(A z_{\text {misaligned congruent }}-A z_{\text {misaligned incongruent }}\right) .
\end{aligned}
$$


Paired-sample t-tests revealed that there was no significant difference between the control condition and either global $\left(\mathrm{t}_{(47)}=0.40, p=0.69, \mathrm{~d}=0.07\right)$ or local $\left(\mathrm{t}_{(47)}=0.21\right.$,

a Perceptual sensitivity with composite faces
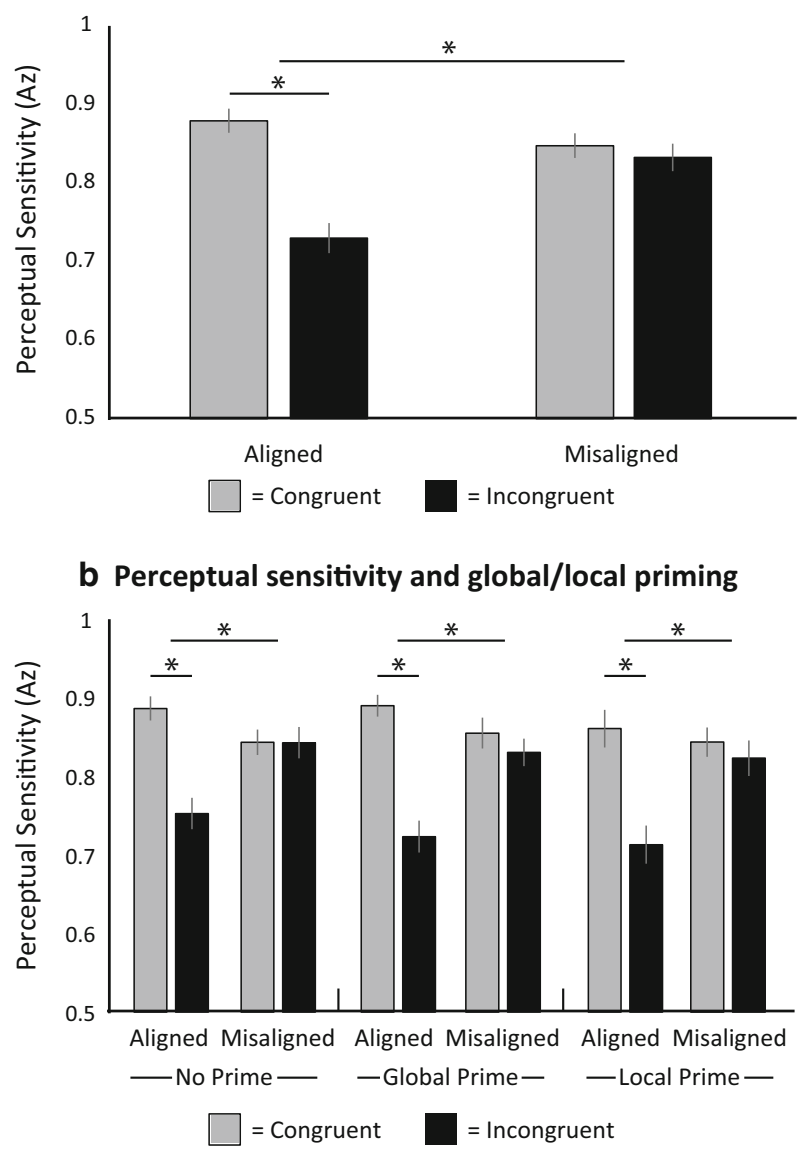

C Composite-face effect strength and global/local priming

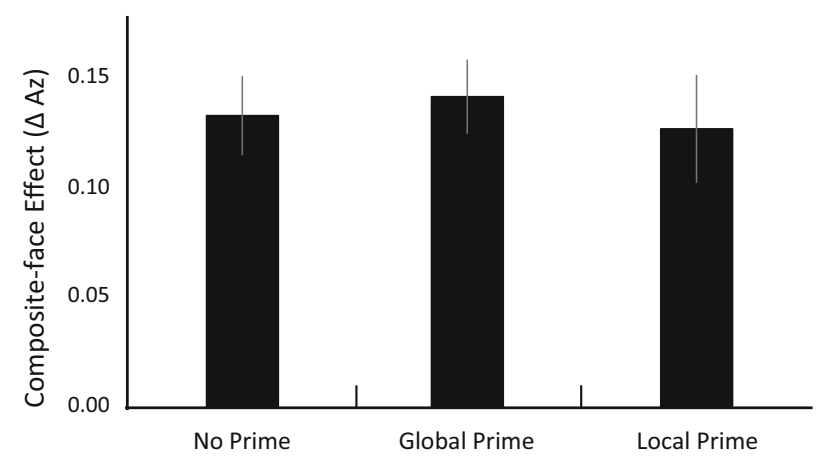

Fig. 3 Perceptual sensitivity. The composite-face effect was observed collapsed across types of attentional scope (a), with a strong effect of congruency for aligned but not misaligned faces. The composite-face effect was seen in all priming conditions (b), but the strength of the effect did not vary between attentional-scope manipulations (c). Asterisk indicates significant differences at the level of $p<0.05$ $p=0.83, \mathrm{~d}=0.04)$ attentional scopes, and importantly, no difference between CFEs under global versus local attentional scopes $\left(\mathrm{t}_{(47)}=0.50, p=0.62, \mathrm{~d}=0.10\right)$ at the group level (Fig. 3c).

\section{Sensitivity to Composite-Face Effect and Autistic Traits}

Next, we investigated whether individual differences in autistic traits were related to attentional scope modulation of the CFE. Specifically, we tested four a priori predictions about the relationship between the strength of the CFE and AQ scores:

1. We predicted that individuals with higher overall $\mathrm{AQ}$ scores, and thus a greater disposition to exhibit ASD traits, would show a weaker CFE.

2. We predicted that hypothesis 1 above would be specifically driven by the Attention to Detail subscore of the AQ, which previous research has suggested may be related to local-processing bias (Stewart and Austin 2009).

3. Based on the hypothesis that individuals with ASD default to local processing but are fully capable of global processing (Mottron et al. 2003, 2006; Plaisted et al. 1999; Tanaka and Sung 2013), we predicted that individuals with higher AQ scores would be more susceptible to attentional-scope manipulations, represented by a stronger CFE in global relative to locally attended conditions ( $\left.\mathrm{CFE}_{\text {global }}-\mathrm{CFE}_{\text {local }} ; \Delta \mathrm{CFE}\right)$.

4. We predicted that the relationship in hypothesis 3 would be driven by the Attention to Detail subscale on the $\mathrm{AQ}$, which we expect to be more related to $\triangle \mathrm{CFE}$ when using a global attentional scope.

To test the first hypothesis, a Pearson correlation was calculated between AQ score and the strength of the CFE in the control condition without attentional scope modulation, revealing no significant relationship $(\mathrm{r}=-0.06$, $p=0.71$ ). To address the second hypothesis, the predicted relationship between the scores on the Attention to Detail subscale of the AQ and the strength of the CFE with no attentional scope modulation was not significant $(\mathrm{r}=-0.06, p=0.71)$. Contrary to the third hypothesis, overall AQ score did not predict $\triangle \mathrm{CFE}$ across global/local instructions $(\mathrm{r}=0.09, p=0.53)$. In line with our fourth prediction, however, the Attention to Detail subscore of the AQ was significantly correlated with $\triangle \mathrm{CFE}$ between global/local attentional scope $(\mathrm{r}=0.31, p=0.03$; Fig. 4a).

We predicted that a local-processing bias is the default attentional scope of individuals with ASD, and thus possibly also in individuals with high levels of ASD traits. As such, a default to local processing should be driven primarily by individuals with high levels of ASD traits 
shifting away from local processing during the global priming condition, resulting in a stronger CFE. To further examine this prediction, follow-up correlations were calculated relating scores on the Attention to Detail subscale to the CFE under the global and local attentional scope manipulations independently. Indeed, the strength of the CFE following "attend global" instructions was significantly correlated with scores on the Attention to Detail subscale (Fig. 4b, $\mathrm{r}=0.34, p=0.02$ ), but not following "attend local" instructions ( $\mathrm{r}=-0.10, p=0.51$; Fig. $4 \mathrm{c})$, suggesting that individuals with higher local-processing bias as measured by the AQ were more susceptible to shifts of their default processing style following global attentional scope manipulations than during local priming (Fig. 4b, c). Importantly, this difference in correlation across global and attentional scopes was significant $(\mathrm{z}=2.16, p=0.03)$.

Finally, the relationship between sensory profile scores and CFE was examined. Given the dearth of empirical studies relating stable sensory preference and perceptual binding, configural processing, or holistic processing, these analyses were exploratory, and findings herein should be treated as preliminary. Following the pattern of analysis above, overall scores on the sensory profile were not correlated with the strength of the CFE in the absence of attentional manipulations, revealing no significant relationship $(\mathrm{r}=0.08, p=0.61)$. A significant relationship between $\triangle$ CFE across global and local attentional scope manipulations and overall sensory profile scores was observed ( $\mathrm{r}=-0.30, p=0.04$; Fig. 5a). Furthermore, differences in correlations between SP scores and global and local attentional scope manipulations were just shy of significant $(\mathrm{z}=1.93, p=0.05)$. This difference was driven by a trending negative correlation between sensory profiles and CFE under global attentional scope (Fig. 5b, $\mathrm{r}=-0.25, p=0.09$ ) relative to a slight positive but not significant correlation between sensory profiles and CFE under local attentional scope (Fig. $6 c, r=0.15, p=0.32$ ).

\section{Criterion with Composite-Face Effect}

As in Gao et al. (2011) previous paper, we also calculated decision criterion (C) separately for each condition in the composite-face task. Decision criterion is a signal detection measure, which indexes the degree of bias in responses, with positive values indicating a bias towards responding "different." An omnibus three-way repeated-measure ANOVA was conducted with factors of attentional scope (control, global, and local), alignment (aligned and misaligned), and congruency (congruent and incongruent). Main effects of alignment $\left(\mathrm{F}_{(1,47)}=39.48, p<0.001\right.$, $\left.\eta_{\mathrm{p}}^{2}=0.46\right)$ and congruency $\left(\mathrm{F}_{(1,47)}=78.13, p<0.001\right.$, $\eta_{\mathrm{p}}^{2}=0.62$ ) were observed, with participants more likely to a Relationship of global/local attentional scope and AQ

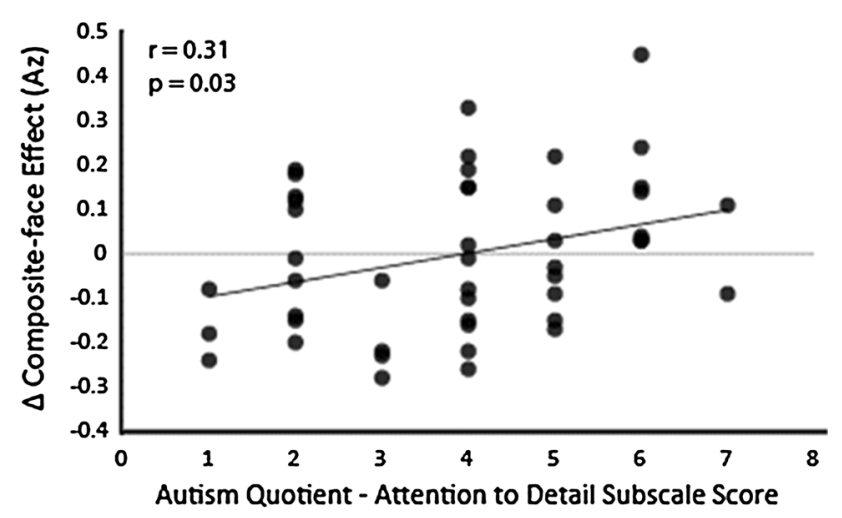

b AQ vs CFE bias with global attentional scope

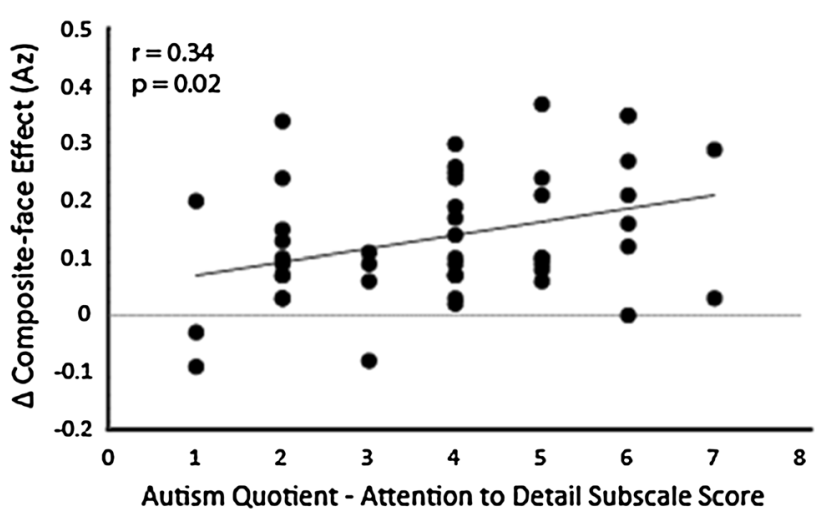

C AQ vs CFE bias with local attentional scope

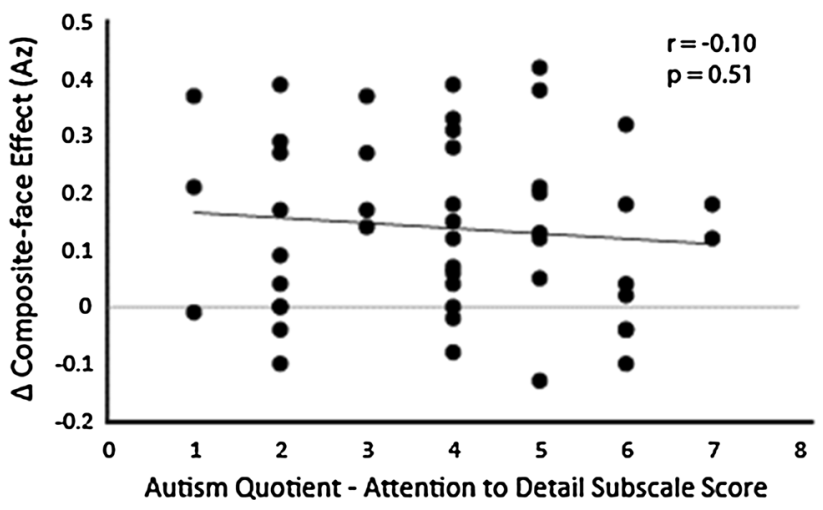

Fig. 4 Relationships with Attention to Detail. The differential response to behavioral global and local attentional scope manipulations $\left(\mathrm{CFE}_{\text {global }}-\mathrm{CFE}_{\text {local }}\right.$; labelled on the y-axes as " $\Delta$ Compositeface Effect") was significantly correlated with the ASD trait of Attention to Detail (a). The stronger the Attention to Detail ASD trait, the more susceptible individuals were to global attentional scope manipulations (b), but no such relationship was seen following local attentional scope manipulations (c). CFE composite-face effect

respond "different" to aligned trials as well as incongruent trials (see Fig. 6). A significant alignment $\mathrm{x}$ congruency interaction was also observed $\left(\mathrm{F}_{(1,47)}=22.17, p<0.001\right.$, $\left.\eta_{\mathrm{p}}^{2}=0.32\right)$. There was no main effect of attentional scope 
a Relationship of global/local priming and SP

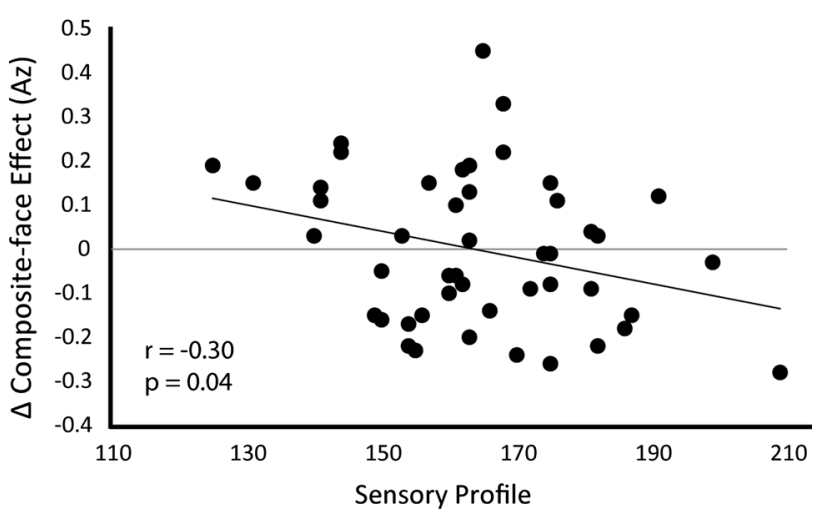

b SP vs local-processing bias following global prime

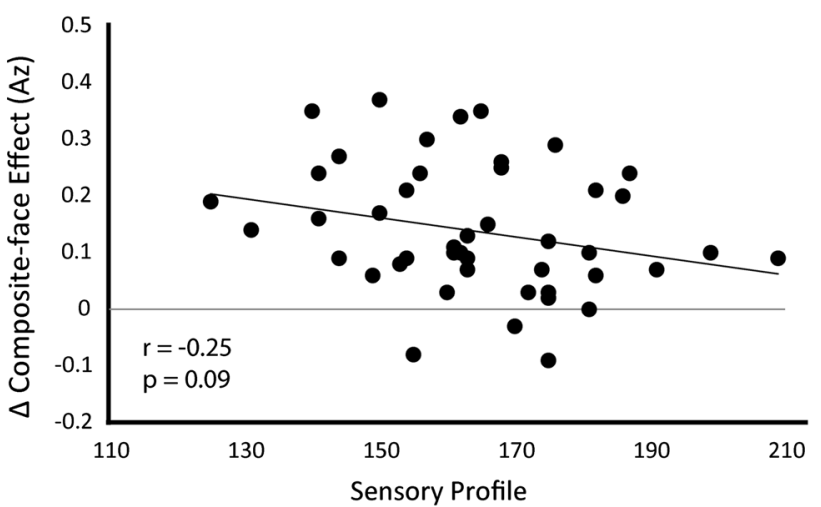

C SP vs local-processing bias following local prime

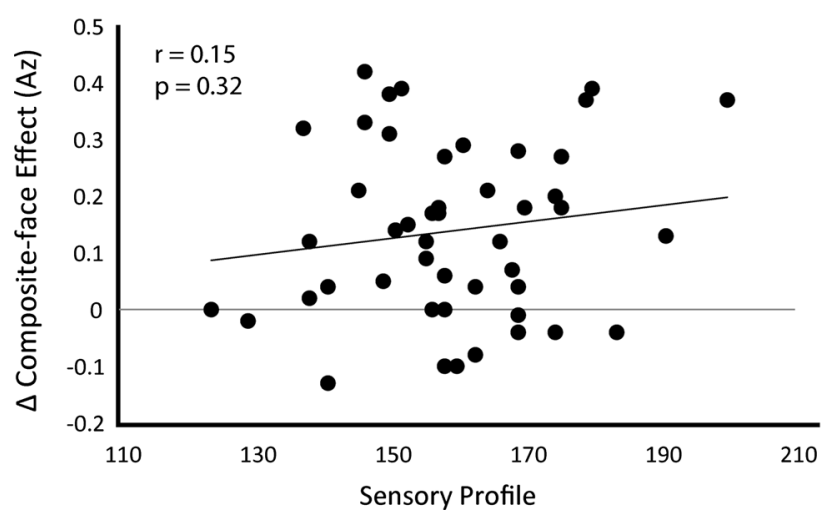

Fig. 5 Relationships with the sensory profile. The differential response to global and local attentional scope manipulations $\left(\mathrm{CFE}_{\text {global }}-\mathrm{CFE}_{\text {local }}\right.$; labelled on the $\mathrm{y}$-axes as " $\Delta$ Composite-face effect") was significantly correlated with sensory profile score (a). The lower the sensory profile score, the more impacted individuals were by adopting a global attentional scope (b), with the reverse pattern observed with a local attentional scope (c). CFE compositeface effect

$\left(\mathrm{F}_{(2,}\right.$ 46) $\left.=0.06, \quad p=0.94, \quad \eta_{\mathrm{p}}^{2}<0.01\right)$, no interaction between attentional scope and alignment $\left(\mathrm{F}_{(2,46)}=1.92\right.$, $\left.p=0.15, \quad \eta_{\mathrm{p}}^{2}=0.04\right)$ or congruency, $\left(\mathrm{F}_{(2,46)}=0.13\right.$, $\left.p=0.88, \eta_{\mathrm{p}}^{2}<0.01\right)$, and no three way interaction

\section{a Decision criterion with composite faces}

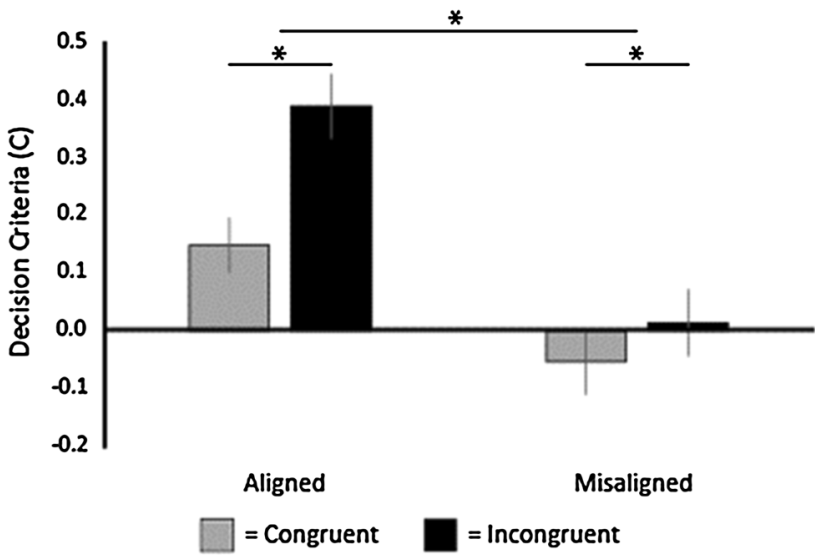

b Decision criterion and global/local attentional scope

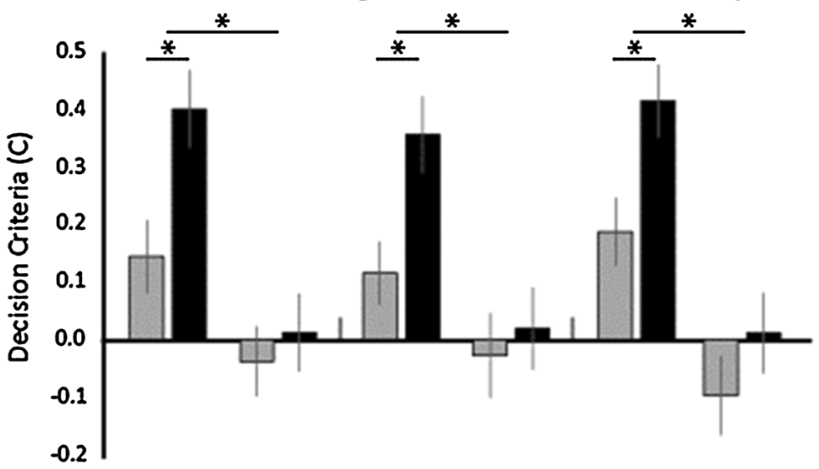

Aligned Misaligned Aligned Misaligned Aligned Misaligned - Control _- - Global Scope - _ L Local Scope-

= Congruent $\square=$ Incongruent

Fig. 6 Decision criterion. Decision criterion, collapsed across attentional scope manipulations (a), showed significantly stronger effects of congruency in the aligned, relative to the misaligned, conditions. This relationship was seen regardless of attentional scope manipulation (b), but was not related to ASD traits or sensory profile scores. Asterisk indicates significant differences at the level of $p<0.05$

$\left(\mathrm{F}_{(2,46)}=0.58, p=0.56, \eta_{\mathrm{p}}^{2}<0.01\right)$. Follow-up tests, collapsing across attentional scope, revealed that the interaction between alignment and congruency was driven by a strong difference in criterion between congruent and incongruent presentations that were aligned, with participants more likely to respond "different" to incongruent trials $\left(\mathrm{t}_{(47)}=9.10, p=6.10 \mathrm{e}^{-12}, \mathrm{~d}=0.67\right)$, relative to a smaller difference when misaligned $\left(\mathrm{t}_{(47)}=2.81\right.$, $p=0.007, \mathrm{~d}=0.17$; Fig. 6a), replicating the findings in Gao et al. (2011).

\section{Criterion with Composite-Face Effect and Autistic Traits}

Since, to our knowledge, no studies as of yet have investigated decision-level processing and ASD traits, we were 
unsure if there would be a relationship between decision criterion on the composite-face task and AQ scores. On the one hand, based on work in which autistic individuals showed less sensitivity to contextual framing effects when making decisions (De Martino et al. 2008), we might expect individuals with high levels of ASD traits to show decreased criterion shifts following attentional scope manipulations than individuals with lower levels of ASD traits. On the other hand, previous work with the CFE showed no impact of decision-level criterion shifts on attentional scope (Gao et al. 2011), suggesting that we might likewise find no relationship when taking ASD traits into account. We thus calculated correlations between the Attention to Detail subscale and shifts in criterion driven by attentional scope to investigate whether the relationship between change in sensitivity $(\mathrm{Az})$ between attentional scope manipulations and autistic traits would also be mirrored in decision processes, indexed by decision criterion.

To explore a possible relationship between criterion and overall AQ score, a Pearson correlation was calculated between AQ score and the average criterion for all conditions following a global $(\mathrm{r}=-0.10, p=0.46)$ and local ( $\mathrm{r}=0.27, p=0.06$ ) attentional scope manipulation, with only the local condition trending towards significance. Furthermore, we also did not observe a relationship between the Attention to Detail subscale and criterion following either global $(\mathrm{r}=-0.14, p=0.33)$ or local ( $\mathrm{r}=0.13, p=0.37$ ) manipulations. We also correlated overall AQ scores with the criterion during the important aligned-incongruent condition. There was no significant relationship with criterion during the aligned-incongruent condition following global $(\mathrm{r}=0.02, p=0.89)$ or local ( $\mathrm{r}=0.25, p=0.09$ ) manipulations, though again the local attentional scope condition was trending towards significance. Nor did we observe a relationship between the $A t$ tention to Detail subscale and aligned-incongruent criterion following either global $(-0.06, p=0.68)$ or local ( $\mathrm{r}=0.19, p=0.20)$ instructions

Finally, the relationship of decision criterion to the sensory profile scores was also measured. No correlation was observed between average criterion and sensory profiles $(\mathrm{r}=0.004, p=0.98)$ or between attentional scope and sensory profiles $(\mathrm{r}=-0.08, p=0.60)$. This is consistent with the idea that decision-level processes, indexed by response criterion, are not related to sensory perception in this sample.

\section{Discussion}

This study investigated how global/local processing styles relate to individual differences in autistic traits. We modulated attentional scope by directing individuals in a composite-letter task to attend either the global or local level of the stimuli. Subsequently, we tested for the effect of this modulation on the composite-face task, a standard test of holistic face processing. Three major findings were evident. First, the Attention to Detail scale of the AQ was significantly related to behavioral effects of attentional scope; individuals with a stronger local-processing bias as measured with the AQ were more susceptible to the influence of global priming, relative to individuals with weaker "default" local bias on the AQ. Second, these results support the hypothesis that the local-processing biases often seen in ASD are an implicit perceptual processing style that does not necessitate a global-processing deficiency. Finally, the relationship between local-processing biases measured with the AQ and attentional scope manipulation manifests in perceptual sensitivity (Az), and was not found in decision criterion (C), measured on the same task. This suggests that local-processing biases influence face perception more at the level of perception rather than at the level of decision-making.

The majority of theoretical accounts of ASD include some aspect of local-processing bias (Frith and Happe 1994; Kanner 1943; Pellicano and Burr 2012), and the literature is replete with examples of enhanced local-processing efficiency in ASD (Bertone et al. 2005; Happé 1996; Jolliffe and Baron-Cohen 1997; Joseph et al. 2009; Kemner et al. 2008; Minshew et al. 1997; O'Riordan and Plaisted 2001; Shah and Frith 1983, 1993). This localprocessing bias can come at the cost of global processing, as proposed by the Weak Central Coherence hypothesis, which suggests that autistic individuals lose the proverbial forest for the sake of the trees (Happe and Frith 2006). Other accounts claim that such a local-processing bias is simply a different default mode of processing. That is, while most individuals' attentional settings default to processing the global percept, autistic individuals tend to default to processing local component percepts (Iarocci et al. 2006). Importantly, this perspective, supported by our data, does not predict a deficit in global processing in ASD or in individuals with high levels of ASD traits. Indeed, such a lack of global-processing deficits has been observed in a number of studies to date (Deruelle et al. 2006; Mottron et al. 2003, 2006; Wang et al. 2004).

Using the composite-letter and composite-face tasks in conjunction, we were able to test the divergent predictions made by these two theories of ASD. The impaired ability to recognize two identical top-halves of faces when they are paired, and aligned with two differing bottom halves, is a strong indicator that an individual perceptually bound the facial features in the top and bottom halves of the face (i.e., holistic or configural processing) and was unable to process them independently. Evidence for such processing has been seen both behaviorally (Gao et al. 2011; Tanaka and Farah 
1993) and electrophysiologically (Jacques and Rossion 2009, 2010; Kuefner et al. 2010; Schiltz et al. 2010). In addition to measuring base levels of holistic processing, the addition of global and local scope manipulations allows us to measure whether individuals are able to successfully change their perceptual strategies and shift their level of either global- or local-processing biases (Gao et al. 2011).

Our data show that individuals with high scores on the Autism Quotient's Attention to Detail subscale were able to shift their processing level from local processing to global processing in a composite-face task when attention was implicitly directed to the global level in a composite-letter task. As such, while these individuals' default processing mode was favouring local processing, they were able to successfully shift their perceptual bias towards global processing. These novel findings converge with previous data in a clinical sample of individuals with ASD (Plaisted et al. 1999). When specifically directed to focus on either the global or local aspects of a stimulus, autistic individuals showed no impairment, but when undirected, autistic individuals were slower to detect the global percept. Our data thus support the hypothesis that autistic individuals and individuals with high levels of ASD traits are fully capable of completing global tasks unimpaired when focusing on the global scope.

This apparent default to local processing, and the ability to successfully shift towards a global processing strategy, was explicitly seen with measures of perceptual sensitivity during face perception. In contrast, no relationship was observed between ASD traits and the impact of global/local attentional scope on the decision criterion, which suggests that the default local bias related to ASD manifests more at a perceptual level, rather than a decision stage of processing. The data suggesting that criterion shifts between individual conditions, specifically the high bias to respond "different" to aligned-incongruent stimuli, successfully replicates previous research (Cheung et al. 2008; Gao et al. 2011), as does the lack of effect of attentional scope (Gao et al. 2011). These current data extend this finding to the novel suggestion that changes in face perception related to the ASD trait of Attention to Detail are also restricted to changes at the perceptual level. This new finding was further supported by the correlational analysis in which criterion did not covary consistently with individuals' sensory preferences, whereas sensitivity shifts did.

While these data strongly suggest that the local-processing bias seen in ASD and individuals with high levels of ASD traits is a default sensory preference without an associated impairment in global processing, we were surprised to find no relationship between local-processing bias and the CFE in the absence of attentional scope manipulations. With that said, while we predicted that individuals with high levels of local-processing bias would be less susceptible to the CFE, our sample was well outside the range of clinical symptomatology for ASD. As such, it is well within reason to consider that the relationship between local- and global-processing biases and the CFE is not linear. That is, given the lack of such a finding in our population without ASD here, coupled with findings in clinical samples of ASD in which a weaker CFE was measured relative to typically developed peers (Gauthier et al. 2009; but see Teunisse and de Gelder 2003), it may be that a local-processing bias influences perception to a greater extent when local-processing biases are at a clinical ASD level, but less so in the typical range.

\section{Broader Impacts of a Local-Processing Default}

Changes in face perception due to atypical global/local processing biases have numerous implications, as face perception contributes to social communications in a wide variety of ways. Besides being able to determine who an individual is at the sight of their face, typical face perception includes processing of emotional expressions (Ekman 1972), contributions to speech perception through the facial articulations of language (Sumby and Pollack 1954), and directed attention via the perception of other's eye gaze for joint attention (Scaife and Bruner 1975), to name a few. Perhaps related to their default towards local processing, autistic individuals exhibit specific difficulties with each of these tasks: facial perception (Behrmann et al. 2006b; Blair et al. 2002; Boucher and Lewis 1992; Gepner et al. 1996; Hauck et al. 1998; Klin et al. 1999; Tantam et al. 1989; Wallace et al. 2008), recognition of social cues (Baron-Cohen et al. 1997; Baron-Cohen et al. 2001a, b) and facial emotional expression (for review, see Harms et al. 2010).

In addition to all of these issues specifically related to facial perception, atypical preferences towards local processing may have even broader impacts. In fact, sensory processing studies in ASD have consistently shown differences in the ability of individuals with ASD to integrate multiple pieces of sensory information into a global, meaningful percept. One example of this in the visual realm is joint attention (for review, see Bruinsma et al. 2004). During joint attention, one individual perceives that a second individual is attending to a particular object in the environment, and subsequently directs his or her attention to that same object. Joint attention requires an individual to perceive the object in the environment, the direction of other individual's eye gaze, and importantly, needs to be able to integrate this information to realize that the object is the focus of their attention. These issues in ASD can also been seen beyond the visual realm, with difficulties in merging pieces of sensory information across sensory modalities into a global, unified percept (Baum et al. 2015; Foss-Feig et al. 2010; Foxe et al. 
2013; Iarocci and McDonald 2006; Kwakye et al. 2011; Stevenson et al. 2014a, b, c, d, 2015; Wallace et al. 2014; Woynaroski et al. 2013). For example, autistic individuals are less able to integrate the auditory and visual components of speech signals and thus exhibit impairments in speech perception (Foxe et al. 2013; Iarocci et al. 2010; Irwin et al. 2011; Smith and Bennetto 2007; Williams et al. 2004).

While these and other impacts of differences in global/ local processing biases may result in socio-communicative difficulties, the finding that a simple perceptual prime can influence an individual with high levels of ASD traits to shift their processing towards a global level suggest that this bias is malleable. This ability to shift towards global processing is promising in terms of mitigating issues that may arise from local-processing biases in autistic individuals.

We have discussed our results primarily in terms of local and global processing as defined as fine- or coarse-grain sensory and attentional processing, respectively, this is not the only possible explanation. The global condition of the Navon letter task places more emphasis on lower spatial frequency information, whereas the local task requires processing of higher spatial frequency information (Badcock et al. 1990). Repeatedly attending to either higher or lower spatial frequency information may result in visual adaptation, resulting in a bias toward the other end of the spatial frequency spectrum during subsequent perception of the composite faces (Hills and Lewis 2009). Furthermore, there is evidence to suggest that holistic face perception seems to rely heavily on low spatial frequency information (Goffaux and Rossion 2006), and spatial frequency bias may be an issue in face-perception in autism (Deruelle et al. 2004). Thus, repeatedly focusing on the high- or lowfrequency information may produce the same results as our previously-defined local and global processing (attentional or sensory bias towards fine- or coarse-grained sensory information, respectively). Our results do not preclude this explanation, nor is it inconsistent with our conclusions. Specifically, if global/local attentional scope is better characterized as simply a bias towards one end of the spatial frequency spectrum, then the influence of such biases that are acquired during exposure to Navon letters on faces is still predicted by individual differences in attention-to-detail. Although this interpretation cannot be specifically tested in this current design, it does, however, warrant further investigation.

\section{Conclusions}

The data presented in this study provide evidence that the local processing biases associated with ASD traits reflect a default cognitive and perceptual style that focuses on local aspects of the environment that is not associated with a concomitant decrease in global-processing abilities. This finding may extend to individuals with a clinical ASD diagnosis, though this needs to be directly tested in future experiments. Furthermore, these data suggest that simple attentional scope manipulations in individuals with high levels of ASD traits can shift processing styles from locally-focused to globally-focused. These effects operate at the perceptual or attentional level, not at the level of decision making as indexed by response criterion, as ASD traits covaried with perceptual sensitivity on the composite-face task, but not decision criterion. This finding was also bolstered by the significant relationship between sensory preference (as measured by the Adult Sensory Profile) and differences in sensitivity between priming conditions, but not response criterion.

Author contribution R.S.-NSERC Banting Postdoctoral Fellowship, CIHR Autism Research Training program; S.S.-NSERC CGS-M Scholarship; J.C.-NSERC Discovery Grant 435647-2013; M.B.Scholar Award from the James S McDonnell Foundation, CIHR Grant MOP-115148; S.F.-NSERC Grant 216203-13, CIHR Grant 106436.

\section{References}

Almeida, R. A., Dickinson, J. E., Maybery, M. T., Badcock, J. C., \& Badcock, D. R. (2014). Enhanced global integration of closed contours in individuals with high levels of autistic-like traits. Vision research, 103, 109-115.

American Psychiatric Association, American Psychiatric Association, DSM-5 Task Force. (2013). Diagnostic and statistical manual of mental disorders: DSM-5, 5th edn. Arlington, VA: American Psychiatric Association.

Association, A. P. (2000). Diagnostic and statistical manual of mental disorders, text revision (DSM-IV-TR). Arlington, VA: American Psychiatric Association.

Badcock, J. C., Whitworth, F. A., Badcock, D. R., \& Lovegrove, W. J. (1990). Low-frequency filtering and the processing of localglobal stimuli. Perception, 19, 617-629.

Baron-Cohen, S., Wheelwright, S., \& Jolliffe, Therese. (1997). Is there a" language of the eyes"? Evidence from normal adults, and adults with autism or Asperger syndrome. Visual Cognition, $4,311-331$.

Baron-Cohen, S., Wheelwright, S., Skinner, R., Martin, J., \& Clubley, E. (2001a). The autism-spectrum quotient (AQ): Evidence from asperger syndrome/high-functioning autism, malesand females, scientists and mathematicians. Journal of Autism and Developmental Disorders, 31, 5-17.

Baron-Cohen, S., Wheelwright, S., Hill, J., Raste, Y., \& Plumb, I. (2001b). The "Reading the Mind in the Eyes" test revised version: A study with normal adults, and adults with Asperger syndrome or high-functioning autism. Journal of Child Psychology and Psychiatry, 42, 241-251.

Baum, S. H., Stevenson, R. A., \& Wallace, M. T. (2015). Behavioral, perceptual, and neural alterations in sensory and multisensory function in autism spectrum disorder. Progress in Neurobiology, 134, 140-160.

Behrmann, M., et al. (2006a). Configural processing in autism and its relationship to face processing. Neuropsychologia, 44, 110-129.

Behrmann, M., Thomas, C., \& Humphreys, K. (2006b). Seeing it differently: Visual processing in autism. Trends in Cognitive Sciences, 10, 258-264. 
Bertone, A., Mottron, L., Jelenic, P., \& Faubert, J. (2005). Enhanced and diminished visuo-spatial information processing in autism depends on stimulus complexity. Brain, 128, 2430-2441. doi:10. 1093/brain/awh561.

Blair, R., Frith, U., Smith, N., Abell, F., \& Cipolotti, L. (2002). Fractionation of visual memory: Agency detection and its impairment in autism. Neuropsychologia, 40, 108-118.

Bölte, S., Holtmann, M., Poustka, F., Scheurich, A., \& Schmidt, L. (2007). Gestalt perception and local-global processing in highfunctioning autism. Journal of Autism and Developmental Disorders, 37, 1493-1504

Boucher, J., \& Lewis, V. (1992). Unfamiliar face recognition in relatively able autistic children. Journal of Child Psychology and Psychiatry, 33, 843-859.

Brown, C., \& Dunn, W. (2002). Adolescent-adult sensory profile: User's manual. Therapy Skill Builders.

Brown, C., Tollefson, N., Dunn, W., Cromwell, R., \& Filion, D. (2001). The adult sensory profile: Measuring patterns of sensory processing. American Journal of Occupational Therapy, 55, $75-82$.

Bruinsma, Y., Koegel, R. L., \& Koegel, L. K. (2004). Joint attention and children with autism: A review of the literature. Mental Retardation and Developmental Disabilities Research Reviews, 10, 169-175.

Busigny, T., \& Rossion, B. (2011). Holistic processing impairment can be restricted to faces in acquired prosopagnosia: Evidence from the global/local Navon effect. Journal of Neuropsychology, $5,1-14$.

Cheung, O. S., Richler, J. J., Palmeri, T. J., \& Gauthier, I. (2008). Revisiting the role of spatial frequencies in the holistic processing of faces. Journal of Experimental Psychology: Human Perception and Performance, 34, 1327.

De Martino, B., Harrison, N. A., Knafo, S., Bird, G., \& Dolan, R. J. (2008). Explaining enhanced logical consistency during decision making in autism. The Journal of Neuroscience, 28, 10746-10750.

Deruelle, C., Rondan, C., Gepner, B., \& Fagot, J. (2006). Processing of compound visual stimuli by children with autism and Asperger syndrome. International Journal of Psychology, 41, 97-106.

Deruelle, C., Rondan, C., Gepner, B., \& Tardif, C. (2004). Spatial frequency and face processing in children with autism and Asperger syndrome. Journal of Autism and Developmental Disorders, 34, 199-210.

Dunn, W., \& Westman, K. (1997). The sensory profile: The performance of a national sample of children without disabilities. American Journal of Occupational Therapy, 51, 25-34.

Ekman, P. (1972). Universals and cultural differences in facial expression of emotion. In: J. Cole (Ed.), Nebraska symposium on motivation, 1971. (pp. 207-283). Lincoln: University of Nebraska Press.

Eriksen, C. W., \& James, J. D. S. (1986). Visual attention within and around the field of focal attention: A zoom lens model. Perception and Psychophysics, 40, 225-240.

Foss-Feig, J. H., et al. (2010). An extended multisensory temporal binding window in autism spectrum disorders. Experimental Brain Research, 203, 381-389. doi:10.1007/s00221-010-2240-4.

Foxe, J. J., et al. (2013). Severe multisensory speech integration deficits in high-functioning school-aged children with autism spectrum disorder $(A S D)$ and their resolution during early adolescence. Cerebral Cortex, bht 213 .

Frith, U., \& Happe, F. (1994). Autism: beyond "theory of mind". Cognition, 50, 115-132.

Gao, Z., Flevaris, A. V., Robertson, L. C., \& Bentin, S. (2011). Priming global and local processing of composite faces: revisiting the processing-bias effect on face perception. Attention, Perception, \& Psychophysics, 73, 1477-1486.

Gauthier, I., Klaiman, C., \& Schultz, R. T. (2009). Face composite effects reveal abnormal face processing in Autism spectrum disorders. Vision Research, 49, 470-478. doi:10.1016/j.visres. 2008.12.007.

Gepner, B., Gelder, B. D., \& Schonen, S. D. (1996). Face processing in autistics: Evidence for a generalised deficit? Child Neuropsychology, 2, 123-139.

Goffaux, V., \& Rossion, B. (2006). Faces are" spatial"-holistic face perception is supported by low spatial frequencies. Journal of Experimental Psychology: Human Perception and Performance, $32,1023$.

Gomot, M., et al. (2006). Change detection in children with autism: an auditory event-related fMRI study. NeuroImage, 29, 475-484.

Gomot, M., Giard, M.-H., Adrien, J.-L., Barthelemy, C., \& Bruneau, N. (2002). Hypersensitivity to acoustic change in children with autism: Electrophysiological evidence of left frontal cortex dysfunctioning. Psychophysiology, 39, 577-584.

Happe, F., \& Frith, U. (2006). The weak coherence account: Detailfocused cognitive style in autism spectrum disorders. Journal of Autism and Developmental Disorders, 36, 5-25. doi:10.1007/ s10803-005-0039-0.

Happé, F. G. (1996). Studying weak central coherence at low levels: Children with autism do not succumb to visual illusions. A research note. Journal of Child Psychology and Psychiatry, 37, 873-877.

Harms, M. B., Martin, A., \& Wallace, G. L. (2010). Facial emotion recognition in autism spectrum disorders: A review of behavioral and neuroimaging studies. Neuropsychology Review, 20, 290-322.

Hauck, M., Fein, D., Maltby, N., Waterhouse, L., \& Feinstein, C. (1998). Memory for faces in children with autism. Child Neuropsychology, 4, 187-198.

Hills, P. J., \& Lewis, M. B. (2009). A spatial frequency account of the detriment that local processing of Navon letters has on face recognition. Journal of Experimental Psychology: Human Perception and Performance, 35, 1427.

Hoekstra, R. A., Bartels, M., Cath, D. C., \& Boomsma, D. I. (2008). Factor structure, reliability and criterion validity of the AutismSpectrum Quotient (AQ): A study in Dutch population and patient groups. Journal of Autism and Developmental Disorders, $38,1555-1566$.

Hughes, H. C., Layton, W. M., Baird, J. C., \& Lester, L. S. (1984). Global precedence in visual pattern recognition. Perception and Psychophysics, 35, 361-371.

Hurst, R., Mitchell, J., Kimbrel, N., Kwapil, T., \& Nelson-Gray, R. (2007). Examination of the reliability and factor structure of the Autism Spectrum Quotient (AQ) in a non-clinical sample. Personality and Individual Differences, 43, 1938-1949.

Iarocci, G., Burack, J. A., Shore, D. I., Mottron, L., \& Enns, J. T. (2006). Global-local visual processing in high functioning children with autism: structural vs. implicit task biases. Journal of Autism and Developmental Disorders, 36, 117-129. doi:10. 1007/s10803-005-0045-2.

Iarocci, G., \& McDonald, J. (2006). Sensory integration and the perceptual experience of persons with autism. Journal of Autism and Developmental Disorders, 36, 77-90. doi:10.1007/s10803005-0044-3.

Iarocci, G., Rombough, A., Yager, J., Weeks, D. J., \& Chua, R. (2010). Visual influences on speech perception in children with autism. Autism, 14, 305-320. doi:10.1177/1362361309353615.

Irwin, J. R., Tornatore, L. A., Brancazio, L., \& Whalen, D. H. (2011). Can children with autism spectrum disorders "hear" a speaking face? Child Development, 82, 1397-1403. doi:10.1111/j.14678624.2011.01619.x. 
Jacques, C., \& Rossion, B. (2009). The initial representation of individual faces in the right occipito-temporal cortex is holistic: Electrophysiological evidence from the composite face illusion. Journal of Vision, 9, 8.

Jacques, C., \& Rossion, B. (2010). Misaligning face halves increases and delays the N170 specifically for upright faces: Implications for the nature of early face representations. Brain Research, 1318, 96-109.

Johnson, S. A., Blaha, L. M., Houpt, J. W., \& Townsend, J. T. (2010). Systems factorial technology provides new insights on globallocal information processing in autism spectrum disorders. Journal of Mathematical Psychology, 54, 53-72. doi:10.1016/j. jmp.2009.06.006.

Jolliffe, T., \& Baron-Cohen, S. (1997). Are people with autism and Asperger syndrome faster than normal on the Embedded Figures Test? Journal of Child Psychology and Psychiatry, 38, 527-534.

Joseph, R. M., Keehn, B., Connolly, C., Wolfe, J. M., \& Horowitz, T. S. (2009). Why is visual search superior in autism spectrum disorder? Developmental science, 12, 1083-1096.

Kanner, L. (1943). Autistic disturbances of affective contact. Nervous Child, 2, 217-250.

Kemner, C., Van Ewijk, L., Van Engeland, H., \& Hooge, I. (2008). Brief report: Eye movements during visual search tasks indicate enhanced stimulus discriminability in subjects with PDD. Journal of Autism and Developmental Disorders, 38, 553-557.

Kenny, L., Hattersley, C., Molins, B., Buckley, C., Povey, C., \& Pellicano, E. (2015). Which terms should be used to describe autism? Perspectives from the UK autism community. Autism. doi: $10.1177 / 1362361315588200$.

Klin, A., Sparrow, S. S., de Bildt, A., Cicchetti, D. V., Cohen, D. J., \& Volkmar, F. R. (1999). A normed study of face recognition in autism and related disorders. Journal of Autism and Developmental Disorders, 29, 499-508.

Koldewyn, K., Jiang, Y. V., Weigelt, S., \& Kanwisher, N. (2013). Global/local processing in autism: Not a disability, but a disinclination. Journal of Autism and Developmental Disorders, $43,2329-2340$.

Kuefner, D., Jacques, C., Prieto, E. A., \& Rossion, B. (2010). Electrophysiological correlates of the composite face illusion: Disentangling perceptual and decisional components of holistic face processing in the human brain. Brain and Cognition, 74, 225-238.

Kwakye, L. D., Foss-Feig, J. H., Cascio, C. J., Stone, W. L., \& Wallace, M. T. (2011). Altered auditory and multisensory temporal processing in autism spectrum disorders. Frontiers in Integrative Neuroscience, 4, 129. doi:10.3389/fnint.2010.00129.

Lowe, M. X., et al. (2015). Sensory processing patterns predict the integration of information held in visual working memory. Journal of Experimental Psychology: Human Perception and Performance. doi:10.1037/xhp0000131.

Maurer, D., Le Grand, R., \& Mondloch, C. J. (2002). The many faces of configural processing. Trends in Cognitive Sciences, 6, 255-260.

Minshew, N. J., Goldstein, G., \& Siegel, D. J. (1997). Neuropsychologic functioning in autism: Profile of a complex information processing disorder. Journal of the International Neuropsychological Society, 3, 303-316.

Mottron, L., Burack, J. A., Iarocci, G., Belleville, S., \& Enns, J. T. (2003). Locally oriented perception with intact global processing among adolescents with high-functioning autism: evidence from multiple paradigms. Journal of Child Psychology and Psychiatry, 44, 904-913.

Mottron, L., Dawson, M., Soulieres, I., Hubert, B., \& Burack, J. (2006). Enhanced perceptual functioning in autism: an update, and eight principles of autistic perception. Journal of Autism and
Developmental Disorders, 36, 27-43. doi:10.1007/s10803-0050040-7.

Müller, N. G., Bartelt, O. A., Donner, T. H., Villringer, A., \& Brandt, S. A. (2003). A physiological correlate of the "zoom lens" of visual attention. The Journal of Neuroscience, 23, 3561-3565.

Navon, D. (1977). Forest before trees: The precedence of global features in visual perception. Cognitive Psychology, 9, 353-383.

Navon, D. (1981). The forest revisited: More on global precedence. Psychological Research, 43, 1-32.

Nishimura, M., Rutherford, M., \& Maurer, D. (2008). Converging evidence of configural processing of faces in high-functioning adults with autism spectrum disorders. Visual Cognition, 16, 859-891.

O'Riordan, M., \& Plaisted, K. (2001). Enhanced discrimination in autism. The Quarterly Journal of Experimental Psychology, 54, 961-979.

O'Riordan, M. A., Plaisted, K. C., Driver, J., \& Baron-Cohen, S. (2001). Superior visual search in autism. Journal of Experimental Psychology: Human Perception and Performance, 27, 719.

O'riordan, M. A. (2004). Superior visual search in adults with autism. Autism, 8, 229-248.

Pellicano, E., \& Burr, D. (2012). When the world becomes 'too real': A Bayesian explanation of autistic perception. Trends in Cognitive Sciences, 16, 504-510.

Pellicano, E., Gibson, L., Maybery, M., Durkin, K., \& Badcock, D. R. (2005). Abnormal global processing along the dorsal visual pathway in autism: A possible mechanism for weak visuospatial coherence? Neuropsychologia, 43, 1044-1053.

Plaisted, K., Swettenham, J., \& Rees, L. (1999). Children with autism show local precedence in a divided attention task and global precedence in a selective attention task. Journal of Child Psychology and Psychiatry, 40, 733-742.

Rauschenberger, R., \& Yantis, S. (2001). Attentional capture by globally defined objects. Perception and Psychophysics, 63, 1250-1261.

Richler, J. J., Tanaka, J. W., Brown, D. D., \& Gauthier, I. (2008). Why does selective attention to parts fail in face processing? Journal of Experimental Psychology. Learning, Memory, and Cognition, 34, 1356.

Rinehart, N. J., Bradshaw, J. L., Moss, S. A., Brereton, A. V., \& Tonge, B. J. (2000). Atypical interference of local detail on global processing in high-functioning autism and Asperger's disorder. Journal of Child Psychology and Psychiatry, 41, 769-778.

Rossion, B. (2013). The composite face illusion: A whole window into our understanding of holistic face perception. Visual Cognition, 21, 139-253.

Scaife, M., \& Bruner, J. S. (1975). The capacity for joint visual attention in the infant. Nature, 253, 265-266.

Schiltz, C., Dricot, L., Goebel, R., \& Rossion, B. (2010). Holistic perception of individual faces in the right middle fusiform gyrus as evidenced by the composite face illusion. Journal of Vision, 10,25

Shah, A., \& Frith, U. (1983). An islet of ability in autistic children: A research note. Journal of Child Psychology and Psychiatry, 24, $613-620$.

Shah, A., \& Frith, U. (1993). Why do autistic individuals show superior performance on the block design task? Journal of Child Psychology and Psychiatry, 34, 1351-1364.

Smith, E. G., \& Bennetto, L. (2007). Audiovisual speech integration and lipreading in autism. Journal of Child Psychology and Psychiatry, 48, 813-821. doi:10.1111/j.1469-7610.2007.01 766.x.

Stevenson, R. A., Segers, M., Ferber, S., Barense, M. D., Camarata, S., \& Wallace, M. T. (2015). Keeping time in the brain: Autism spectrum disorder and audiovisual temporal processing. Autism Research. doi:10.1002/aur.1566. 
Stevenson, R. A., Segers, M., Ferber, S., Barense, M. D., \& Wallace, M. T. (2014a). The impact of multisensory integration deficits on speech perception in children with autism spectrum disorders. Front Psychol, 5, 379. doi:10.3389/fpsyg.2014.00379.

Stevenson, R. A., et al. (2014b). Multisensory temporal integration in autism spectrum disorders. Journal of Neuroscience, 34, 691-697. doi:10.1523/JNEUROSCI.3615-13.2014.

Stevenson, R. A., et al. (2014c). Brief report: Arrested development of audiovisual speech perception in autism spectrum disorders. Journal of Autism and Developmental Disorders, 44, 1470-1477. doi:10.1007/s10803-013-1992-7.

Stevenson, R. A., et al. (2014d). Evidence for diminished multisensory integration in autism spectrum disorders. Journal of Autism and Developmental Disorders, 44, 3161-3167. doi:10.1007/ s10803-014-2179-6.

Stewart, M. E., \& Austin, E. J. (2009). The structure of the AutismSpectrum Quotient (AQ): Evidence from a student sample in Scotland. Personality and Individual Differences, 47, 224-228.

Sumby, W. H., \& Pollack, I. (1954). Visual contribution to speech intelligibility in noise. Journal of the Acoustical Society of America, 26, 212-215.

Tanaka, J. W., \& Farah, M. J. (1993). Parts and wholes in face recognition. The Quarterly Journal of Experimental Psychology, $46,225-245$

Tanaka, J. W., \& Sung, A. (2013). The "eye avoidance" hypothesis of autism face processing. Journal of Autism and Developmental Disorders. doi:10.1007/s10803-013-1976-7.

Tantam, D., Monaghan, L., Nicholson, H., \& Stirling, J. (1989). Autistic children's ability to interpret faces: A research note. Journal of Child Psychology and Psychiatry, 30, 623-630.

Teunisse, J.-P., \& de Gelder, B. (2003). Face processing in adolescents with autistic disorder: The inversion and composite effects. Brain and Cognition, 52, 285-294.
Van der Hallen, R., Evers, K., Brewaeys, K., Van den Noortgate, W., \& Wagemans, J. (2014). Global processing takes time: A metaanalysis on local-global visual processing in ASD. Psychological bulletin, 141, 549-573. doi:10.1037/bul0000004.

Verde, M. F., Macmillan, N. A., \& Rotello, C. M. (2006). Measures of sensitivity based on a single hit rate and false alarm rate: The accuracy, precision, and robustness of ${ }^{\prime}, \mathrm{A} z$, and A'. Perception and Psychophysics, 68, 643-654.

Wallace, S., Coleman, M., \& Bailey, A. (2008). An investigation of basic facial expression recognition in autism spectrum disorders. Cognition and Emotion, 22, 1353-1380.

Wallace, M. T., \& Stevenson, R. A. (2014). The construct of the multisensory temporal binding window and its dysregulation in developmental disabilities. Neuropsychologia, 64, 105-123.

Wang, A. T., Dapretto, M., Hariri, A. R., Sigman, M., \& Bookheimer, S. Y. (2004). Neural correlates of facial affect processing in children and adolescents with autism spectrum disorder. Journal of the American Academy of Child and Adolescent Psychiatry, 43, 481-490.

Williams, J. H., Massaro, D. W., Peel, N. J., Bosseler, A., \& Suddendorf, T. (2004). Visual-auditory integration during speech imitation in autism. Research in Developmental Disabilities, 25, 559-575. doi:10.1016/j.ridd.2004.01.008.

Woynaroski, T. G., Kwakye, L. D., Foss-Feig, J. H., Stevenson, R. A., Stone, W. L., \& Wallace, M. T. (2013). Multisensory speech perception in children with autism spectrum disorders. Journal of Autism and Developmental Disorders. doi:10.1007/s10803013-1836-5.

Young, A. W., Hellawell, D., \& Hay, D. C. (1987). Configurational information in face perception. Perception, 16, 747-759. 